\title{
Patología dual: situación actual y retos de futuro
}

\author{
Marta TorRens MèLICH \\ Directora de la Línea de Adicciones. Institut d'Atenció Psiquiàtrica, Salut Mental i Addiccions (IAPS)-Hospital del Mar, \\ Universitat Autònoma Barcelona. \\ Enviar correspondencia a: \\ Marta Torrens Mèlich. Institut d'Atenció Psiquiàtrica, Salut Mental i Addiccions (IAPS)-Hospital del Mar, \\ Passeig Marítim 25-29. 08003 Barcelona.Correo e.: mtorrens@imas.imim.es
}

\section{RESUMEN}

Se denomina "patología dual" a la concurrencia en un mismo individuo de, por lo menos, un trastorno por consumo de sustancias y otro trastorno psiquiátrico. Estos pacientes "duales" o con comorbilidad psiquiátrica, son frecuentes y presentan mayor gravedad tanto desde la perspectiva clínica como social que los sujetos que sólo presentan un tipo de trastorno (adictivo u otra enfermedad psiquiátrica). Se presenta un estado de la situación de la patología dual desde la perspectiva epidemiológica, clínica y terapéutica y se revisan los retos de futuro más relevantes. En los retos de futuro se hace hincapié en las necesidades de profundizar en la caracterización nosológica adecuada de la coexistencia de síntomas psicopatológicos y consumo/intoxicación/abstinencia de sustancias; la importancia de disponer de instrumentos clínicos de cribado para detectar la patología dual; la necesidad de desarrollar estrategias terapéuticas farmacológicas y psicológicas efectivas; la importancia de la atención integrada de estos pacientes por un solo equipo terapéutico en una red única de salud mental y adicciones y finalmente, la necesidad de incorporar la formación en patología dual a los profesionales sanitarios.

Palabras clave: patología dual, comorbilidad psiquiátrica, drogodependencias, adicciones.

\section{ABSTRACT}

The term "dual diagnosis" refers to the co-occurrence of at least one substance use disorder with another psychiatric disorder in the same individual. These "dual" patients or patients with psychiatric comorbidity are common, and present greater severity from both the clinical and social perspectives than those subjects with only one type of disorder (addictive or other psychiatric illness). We present the current situation regarding dual diagnosis from epidemiological, clinical and therapeutic perspectives and we review the most important challenges for the future. Among the challenges for the future, we highlight: the need to improve the understanding of the nosological characterisation of the co-occurrence of psychopathological symptoms and the use, intoxication and withdrawal from substances; the need for clinical screening instruments to detect dual diagnosis; the need to develop effective therapeutic strategies both pharmacological and psychological; the importance of providing integrated care to these patients by one therapeutic team within a single mental health and addiction network; and finally, the need to incorporate training on dual diagnosis for health professionals.

Key words: dual diagnosis, psychiatric comorbidity, psychiatric co-occurrence, substance use disorder, addiction.

\section{PATOLOGÍA DUAL: SITUACIÓN ACTUAL}

E los últimos años está de actualidad la llamada "patología dual." Con este término se denomina en muchos ámbitos a la concurrencia en un mismo individuo de, por lo menos, un trastorno por consumo de sustancias y otro trastorno psiquiátrico (por ejemplo la depresión mayor). Estos pacientes "duales" son pacientes especialmente graves tanto desde la perspectiva clínica como social y constituyen un reto terapéutico no sólo a título individual, sino también para los sistemas sanitarios, que deben hacer frente a pacientes complejos a caballo de dos redes asistenciales diferenciadas: la red de atención a drogodependencias y la red de salud mental.

Estas personas con coexistencia de enfermedad adictiva y otras patologías mentales acuden más a los servicios de urgencias y requieren más hospitalizaciones psiquiátricas ${ }^{1,2}$, muestran más conductas de riesgo e infecciones relacionadas como la infección por el VIH y el virus de la hepatitis $C^{3-5}$, mayores tasas 
de desempleo y marginación ${ }^{6,7}$ y más conductas violentas y criminales ${ }^{8,9}$ que los que sólo tienen diagnóstico de drogodependencias o diagnóstico de otro trastorno psiquiátrico. Es decir, se trata de individuos graves desde la perspectiva psicopatológica, médica y social.

Esta gravedad clínica y social es importante porque la patología dual no es una condición aislada sino que tiene una presencia epidemiológica importante. Tanto los estudios llevados cabo en población general como los llevados a cabo en muestras clínicas refieren que la prevalencia de la concurrencia de trastornos por consumo de sustancias y otros trastornos psiquiátricos es elevada, situándose entre el 15-80\% 10-14. Este amplio rango se debe a distintos factores, entre los que cabe destacar: 1) la heterogeneidad de las muestras en las que se ha llevado el estudio: población general, personas que acuden en demanda de tratamiento, consumidores reclutados fuera de los servicios asistenciales; 2) el lugar en el que son atendidos: centros de la red de drogodependencias; recursos asistenciales de la red de salud mental; centros penitenciarios, etc...; 3) factores relacionados con la sustancia de abuso, tanto por sus efectos farmacológicos sobre el sistema nervioso central (sedantes, estimulantes, psicodèlicos), como por su situación legal, disponibilidad y accesibilidad (cuánto más accesible sea una sustancia, más posibilidades hay de que personas sin antecedentes psiquiátricos y con estilos de vida normalizados la consuman); 4) factores intercurrentes como, por ejemplo, la presencia de infección por el VIH que a su vez también se asocia a psicopatología; y 5) las dificultades diagnósticas, que como se describe a continuación, plantean problemas importantes y han sufrido cambios en los criterios utilizados a lo largo del tiempo.

La identificación de forma fiable y válida de un diagnóstico de comorbilidad psiquiátrica en sujetos que están consumiendo sustancias psicoactivas plantea dos problemas fundamentales. Por un lado, el hecho de que los efectos agudos y crónicos de las drogas sobre el sistema nervioso central, simulan síntomas de muchos de los trastornos mentales (por ejemplo, el insomnio producido por el consumo agudo de cocaína, indistinguible del insomnio de enfermedades psiquiátricas como la depresión), dificultando la diferenciación entre los síntomas psicopatológicos de los efectos agudos del consumo o de la abstinencia de la sustancia, de los propios de un trastorno psiquiátrico independiente. Por otro lado, el hecho de que los trastornos psiquiátricos son más "síndromes" (patrones de síntomas con algunas pruebas de validez clínica) que "enfermedades" con una fisiopatología conocida y con unos marcadores biológicos claros que sirvan como "patrón oro" para validar los diagnósticos.

En este sentido, la psiquiatría ha evolucionado con respecto a los conceptos y criterios diagnósticos entre el consumo de drogas y la presencia de otros síntomas psiquiátricos concomitantes. Así desde los criterios de Feighner en $1972^{15}$ hasta los criterios del DSM-IV-TR ${ }^{16}$ y la $\mathrm{CIE}-10^{17}$ se han realizado cambios sustanciales que se han traducido en cambios relevantes en los criterios y en los instrumentos utilizados para realizar los diagnósticos ${ }^{18}$. Los criterios del DSMIV-TR permiten distinguir entre: "trastornos primarios" (trastornos mentales que no son relacionados por el consumo de sustancias ni debidos a una enfermedad médica), "efectos esperados" (síntomas considerados habituales que aparecen como consecuencia del consumo o la abstinencia de una sustancia) o "trastornos inducidos por sustancias" (cuando los síntomas son considerados como excesivos en relación con los que suelen aparecer en los síndromes de intoxicación por o abstinencia a una sustancia), con la finalidad de poder facilitar un diagnóstico más preciso. En este sentido, la entrevista "Psychiatric Research Interview for Substance and Mental Disorders (PRISM) ${ }^{19}$, 20 especialmente diseñada para realizar el diagnóstico de comorbilidad en personas consumidoras de sustancias, ha mostrado buenos índices de validez y fiabilidad en los diagnósticos que establece.

Hay dos hipótesis principales que explican la comorbilidad: 1) la adicción y los otros trastornos psiquiátricos son expresiones sintomáticas distintas de anomalías neurobiológicas preexistentes similares y 2) la administración repetida de drogas, a través de mecanismos de neuroadaptación, origina cambios neurobiológicos que tienen elementos comunes con las anomalías que median ciertos trastornos psiquiátricos. En los últimos años, se han postulado los efectos neurobiológicos del estrés crónico como el puente de unión entre los TUS y las otras enfermedades mentales ${ }^{21}$.

Así pues, la naturaleza de la relación entre los trastornos psiquiátricos y los trastornos por consumo es compleja y puede variar dependiendo de cada trastorno en particular. En general, estos trastornos duales son reflejo de al menos cuatro posibilidades: 1) Las distintas combinaciones de trastornos por uso de sustancias (TUS) y otros trastornos psiquiátricos pueden representar dos o más condiciones independientes, con sus respectivos cursos clínicos y necesidades de tratamiento. Esta combinación puede ocurrir por "casualidad" o como consecuencia de compartir los mismos factores predisponentes (ej. estrés, personalidad, factores ambientales en la infancia, vulnerabilidad genéticas, alteraciones neurobiológicas). 2) El primer trastorno puede influir en el desarrollo de un segundo trastorno, de forma que éste siga un curso independiente. Así el consumo de drogas puede precipitar una predisposición a psicosis (por ejemplo, el consumo de cannabis como factor precipitante para un trastorno esquizofrénico) o causar un cambio fisiológico permanente que origine un trastorno permanente (por ejemplo, el consumo de estimulantes puede originar 
depresión o psicosis). Viceversa, durante la enfermedad psiquiátrica se puede desencadenar una conducta de consumo que derive en un TUS que evolucione de forma independiente (por ejemplo, el consumo de alcohol en episodios maníacos, puede derivar en un alcoholismo independiente). En este caso también ambos trastornos deberán tratarse adecuadamente y durante tanto tiempo como se requiera. 3) El consumo de sustancias puede paliar la sintomatología psiquiátrica de un trastorno que no es diagnosticado o tratado convenientemente. En este caso el tratamiento adecuado de la enfermedad psiquiátrica podría hacer desaparecer el TUS. 4) Algunos síndromes pueden ser cuadros psiquiátricos temporales, como psicosis parecidas a esquizofrenia, como consecuencia de intoxicaciones con tipos específicos de sustancias (por ejemplo, psicosis en intoxicaciones por estimulantes) o abstinencia (por ejemplo, depresión en la abstinencia de estimulantes).

A pesar de la relevancia de llevar a cabo tratamientos efectivos en los casos de patología dual, hasta la actualidad este es uno de los puntos más conflictivos y donde, como se comentará posteriormente, existe mayor reto para el futuro.

\section{PATOLOGÍA DUAL: RETOS DE FUTURO}

La elevada gravedad clínica y social junto con sus altas prevalencias y las dificultades terapéuticas, justifica el interés que estos momentos plantea el estudio de la co-ocurrencia de trastornos por consumo de sustancias y otras enfermedades psiquiátricas, tanto desde el conocimiento de sus bases neurobiológicas y su caracterización clínica y diagnóstica, al desarrollo de tratamientos adecuados farmacológicos y psicosociales, así como en la adaptación de los servicios asistenciales.

Así pues, más allá de la necesidad de seguir investigando en las bases neurobiológicas de la patología dual, que sin duda aportarán una mayor comprensión tanto de la enfermedad adictiva así como de los otros trastornos mentales, hay otros retos igualmente relevantes tanto desde la perspectiva nosológica y clínica como terapéutica. A continuación se resumen los principales retos de futuro:

1. Seguir avanzando en la caracterización nosológica adecuada de la coexistencia de síntomas psicopatológicos y consumo/intoxicación/abstinencia de sustancias de abuso. En este sentido, se plantea la necesidad de clarificar esta relación en la elaboración de los criterios diagnósticos del DSM-V. Algunos investigadores apoyan cambiar la terminología actual y sustituir la terminología "trastorno primario" por "trastorno independiente" para enfatizar en la no relación de causalidad ni temporal entre los trastornos. También en los "trastornos inducidos" se plantea la necesidad de una mayor concreción en los criterios temporales, e incluso una sustitución del término "inducido" por el de "trastorno relacionado con la sustancia" para enfatizar la asociación directa entere el consumo (intoxicación o abstinencia) y la aparición de la psicopatología ${ }^{22}$. Sin duda, el hallazgo de marcadores biológicos que pudieran validar los criterios utilizados para definir y diferenciar estas condiciones (independiente o inducido), constituye un importante reto, aunque en la actualidad no se vislumbra que pueda alcanzarse en un futuro inmediato.

2. La necesidad de disponer de instrumentos clínicos cribado válidos y fiables que permitan detectar la presencia de otros trastornos psiquiátricos en personas con TUS.

3. La necesidad de proporcionar terapias efectivas para los pacientes duales. Este punto, constituye un importante reto: cómo deben tratarse (abordajes farmacológicos y psicológicos) y dónde debe llevarse a cabo el tratamiento (centros de salud mental, centros de adicciones)

Hay importantes lagunas sobre las características más adecuadas de los tratamientos que deben recibir estos pacientes, tanto farmacológicos como psicosociales. En cuanto a la psicofarmacología hay que recordar que la mayoría de las veces se aplican tratamientos farmacológicos que han sido estudiados en pacientes psiquiátricos en los que se excluyeron aquellos que consumían sustancias de abuso, o en pacientes drogodependientes que no presentaban otra patología psiquiátrica. Ello conlleva un mayor riesgo de interacciones y problemas de seguridad, tolerabilidad y efectos adversos, entre ellos el potencial de abuso y el agravamiento de la enfermedad psiquiátrica o de la enfermedad adictiva $^{23,24}$. También en los tratamientos psicosociales es necesario avanzar en conocimiento de cuáles son las terapias más eficaces en estos pacientes duales. Estrategias de confrontación que se han visto útiles en el tratamiento de las conductas adictivas, pueden ser menos eficaces e incluso contraproducentes cuando el paciente presenta además otra enfermedad psiquiátrica. Por el contrario, la terapia grupal, la terapia de contingencias y los tratamientos residenciales parecen ser útiles en estos pacientes duales ${ }^{25}$. Así pues es necesario desarrollar terapias específicas dirigidas al paciente dual, que contesten las lagunas en eficacia, tolerabilidad y efectos adversos que tenemos en la actualidad. 
En cuanto a dónde deben ser tratados estos pacientes, los estudios previos apuntan claramente que, para obtener resultados eficientes, el paciente con patología dual debe ser atendido en un sistema integrado, es decir, en un sistema que garantice el abordaje de la adicción y la otra patología psiquiátrica de forma conjunta y simultánea y, además, a largo plazo $^{26}$. Esto implica la necesidad de tener una red única de atención a todos los problemas mentales, incluyendo las adicciones como problemas mentales tal y como ya está recogido en todos los sistemas de clasificación nosológicos de las enfermedades (CIE, DSM). Esta evidencia científica contrasta con la realidad, en la mayoría de los países, de dos redes asistenciales diferenciadas y desafortunadamente, en muchos casos excluyentes, con el riesgo de disminuir la calidad de la asistencia y lo que es aún más peligroso, de que algunos de estos pacientes, sobre todo los más graves (por ejemplo, pacientes psicóticos con dependencia de cocaína) queden excluidos de la asistencia por las dificultades de ser atendidos en una de las redes asistenciales y presentar un trastorno que debe ser atendido en la otra red.

4. Finalmente, los profesionales que asisten a estos pacientes deben tener formación y conocimientos para detectar y tratarlos adecuadamente. En este sentido es importante la formación de todos los profesionales sanitarios implicados en el ámbito de la salud mental en el conocimiento y manejo de la patología dual, con especial énfasis en los profesionales en periodo de formación (por ejemplo, Médicos Internos Residentes, Psicólogos Internos Residentes, Enfermeros Internos Residentes, etc...).

Así pues, la concurrencia de patología adictiva y otra patología psiquiátrica constituye uno de los desafíos más interesantes para los próximos años, no sólo desde la perspectiva de la investigación de los mecanismos neurobiológicos subyacentes a tal coexistencia, con lo que implica de profundización y mejora del conocimiento de la enfermedad mental en general, sino también en el desarrollo de estrategias terapéuticas adecuadas en recursos asistenciales adaptados y por profesionales entrenados convenientemente. Todo un reto para los planificadores de políticas sanitarias y los profesionales de la salud mental.

\section{REFERENCIAS}

1. Martín-Santos R, Fonseca F, Domingo-Salvany A, Ginés $J M$, Imaz ML, Navinés R, et al. Dual diagnosis in the psychiatric emergency room in Spain. European $\mathrm{J}$ Psychiatry 2006; 20:147-156.
2. Aharonovich E, Liu X, Nunes E, Hasin DS. Suicide attempts in substance abusers: effects of major depression in relation to substance use disorders. Am J Psychiatry 2002; 159:1600-1602.

3. King VL, Kidorf MS, Stoller KB, Brooner RK. Influence of psychiatric comorbidity on HIV risk behaviors: changes during drug abuse treatment. J Addict Dis 2000; 19: 65-83.

4. Carey MP, Carey KB, Maisto SA, Gordon CM, Vanable PA. Prevalence and correlates of sexual activity and HIVrelated risk behaviour among psychiatric outpatients. J Consult Clin Psychol 2001; 69: 846-850.

5. Rosenberg SD, Goodman LA, Osher FC, Swartz MS, Essock SM, Butterfield MI, et al. Prevalence of HIV, hepatitis $B$, and hepatitis $C$ in people with severe mental illness. Am J Public Health 2001; 91: 31-37.

6. Caton CL, Shrout PE, Eagle PF, Opler LA, Felix A, Dominguez B. Risk factors for homelessness among schizophrenic men: a case-control study. Am J Public Health 1994; 84: 265-270.

7. Vazquez C, Munoz M, Sanz J. Lifetime and 12-month prevalence of DSM-III-R mental disorders among the homeless in Madrid: a European study using the CIDI. Acta Psychiatr Scand 1997; 95: 523-530.

8. Abram KM, Teplin LA. Co-occurring disorders among mentally ill jail detainees: implications for public policy. Am Psyhologist. 1991; 4: 1036-1045.

9. Cuffel B, Shumway M, Chouljian T. A longitudinal study of substance use and community violence in schizophrenia. J Nerv Mental Dis, 1994; 182: 704-708.

10. Flynn PK, Brown BS. Co-occurring disorders in substance abuse treatments: Issues and prospects. J Subst Abuse Treat 2008; 34: 36-47.

11. Rodriguez-Llena MC, Domingo-Salvany A, Brugal MT, Silva TC, Sánchez-Nubió A, Torrens M. Psychiatric comorbidity in young heroin users. Drug Alcohol Depend 2006; 84: 48-55.

12. Nocon A, Bergé D, Astals M, Martín-Santos R, Torrens M. Dual diagnosis in an inpatient drug-abuse detoxification unit. Eur Addict Res 2007; 13:192-200.

13. Herrero MJ, Domingo-Salvany A, Torrens M, Brugal MT; ITINERE Investigators. Psychiatric comorbidity in young cocaine users: induced versus independent disorders. Addiction. 2008; 103:284-93.

14. Casas M, Gual A. Patología psiquiátrica asociada al alcoholismo. Adicciones 2002; 14 (Supl 1): 195-219.

15. Feighner JP, Robins E, Guze SB, Woodruff RA Jr, Winokur G, Munoz R. Diagnostic criteria for use in psychiatric research. Arch Gen Psychiatry 1972; 26 : 57-63.

16. American Psychiatric Association. Diagnostic and Statistical Manual of Mental Disorders. 4th ed -TR. Washington, DC: American Psychiatric Press, 2000.

17. Organización Mundial de la Salud. Décima Revisión de la Clasificación Internacional de las Enfermedades. Trastornos mentales del comportamiento y desarrollo. Descripciones clínicas y pautas para el diagnóstico. Madrid: Meditor, 1992. 
18. Torrens M, Martin-Santos R, Samet S. Importance of clinical diagnoses for comorbidity studies in substance use disorders. Neurotox Res 2006;10:253-61.

19. Torrens M, Serrano D, Astals M, Pérez-Domínguez G, Martín-Santos R. Diagnosing Psychiatric Comorbidity in Substance Abusers. Validity of the Spanish Versions of Psychiatric Research Interview for Substance and Mental Disorders (PRISM-IV) and the Structured Clinical Interview for DSM-IV (SCID-IV). Am J Psychiatry. 2004; 161: 1231-1237.

20. Hasin DS, Samet S, Nunes E, Meydan J, Matseoane K, Waxman R. Diagnosis of comorbid psychiatric disorders in substance users assessed with the Psychiatric Research Interview for Substance and Mental Disorders for DSM-IV. Am J Psychiatry 2006; 163: 689-96.

21. Brady KT, Sinha R. Co-occurring mental and substance use disorders: the neurobiological effects of chronic stress. Am J Psychiatry 2005; 162:1483-93.

22. Saunder JB, Schuckit MA, Sirovatka PJ, Regier Da (Eds.). Diagnostic issues in substance use disorders.
Refining the research agenda fro DSM-V. Arlington, American Psychiatric Press, 2007.

23. Mateu G, Astals M, Torrens M. Comorbilidad psiquiátrica y trastorno por dependencia de opiáceos: del diagnóstico al tratamiento. Adicciones 2005; 17 (Supl 2): 111-121.

24. Green Al, Noordsy DL, Brunette MF, O'Keefe C. Substance abuse and schizophrenia: Pharmacotherapeutic intervention. J Subst Abuse Treat 2008; 34: 61-71.

25. Drake RE, O'Neal EL, Wallach MA. A systematic review of psychosocial research on psychosocial interventions for people with co-occurring severe mental and substance use disorders. J Subst Abuse Treat 2008; 34: 123-128.

26. Drake RE, Wallach MA, McGovern MP. Future directions in preventing relapse to substance abuse among clients with severe mental illness. Psychiatric Services, 2005; 56: 1297-1302. 
